\title{
Assessment of the Applicability of Integrative Tumor Response Assays in Advanced Epithelial Ovarian Cancer
}

\author{
JU-HYUN KIM ${ }^{1}$, YONG SIK YOON ${ }^{2}$, JIN CHEON KIM ${ }^{2}$ and YONG-MAN KIM ${ }^{1}$ \\ ${ }^{1}$ Department of Obstetrics and Gynecology, University of Ulsan College of Medicine, \\ Asan Medical Center, Seoul, Republic of Korea; \\ ${ }^{2}$ Department of Surgery, University of Ulsan College of Medicine, Asan Medical Center, Seoul, Republic of Korea
}

\begin{abstract}
Aim: To prospectively correlate clinical responses to second-line chemotherapy for recurrent epithelial ovarian cancer (EOC) with in vitro integrative tumor-response assay (ITRA) results. Patients and Methods: Forty-four patients with advanced EOC were enrolled from 2015-2017 at the Asan Medical Center, Seoul, Korea. ITRA comprised of two sequential histoculture drug response assays (HDRAs) of the tumor tissues. The first stage was HDRA with paclitaxelcarboplatin, paclitaxel, and carboplatin chemotherapy. The second stage was performed with surviving tumor cells from the first stage using topotecan, belotecan, gemcitabine, doxorubicin, ifosfamide, vinorelbine, and etoposide. Results: The median follow-up period was 23.35 (range=4-35.35) months. Eighteen patients (40.9\%) completed the second-line chemotherapy, based on the ITRA results. The objective response rate was $38.9 \%$. The clinical response rate was $50 \%$; two patients $(11.1 \%)$ had stable disease. The sensitivity of ITRA for predicting response was $85.7 \%$ (specificity $=18.2 \%$; accuracy $=44.44 \%)$. Conclusion: ITRAs had acceptable applicability and may help choose second-line chemotherapy for patients with advanced EOC.
\end{abstract}

Epithelial ovarian cancer (EOC) is one of the most challenging and lethal gynecological malignancies $(1,2)$ with a 5-year survival rate below $45 \%$ (3). The current standard of care for patients with advanced EOC is debulking surgery combined with paclitaxel and carboplatin

This article is freely accessible online.

Correspondence to: Professor Yong-Man Kim, MD, Ph.D., Department of Obstetrics and Gynecology, University of Ulsan College of Medicine, Asan Medical Center, 88 Olympic-ro, 43-gil, Songpa-gu, Seoul 05505, Republic of Korea. Tel.: +82 230103640, Fax: +82 24767331, e-mail: ymkim@amc.seoul.kr

Key Words: Epithelial ovarian cancer, tumor response assay, chemotherapy, histoculture drug response assay. chemotherapy (4). However, although patients have a good response to the initial treatment, most tend to experience relapse and develop resistance to platinum-based chemotherapy (5-7). Since platinum plays an essential role in the standard therapy for EOC, the development of platinum resistance implies a poor prognosis (8).

Anticancer therapy for recurrent ovarian cancer (ROC) is chosen based on platinum sensitivity (9). The standard of care for patients with platinum-sensitive ROC is platinum-based chemotherapy (10). In patients with platinum-resistant ROC, non-platinum-based agents, such as pegylated liposomal doxorubicin, topotecan, gemcitabine, and etoposide are preferred, which have similar clinical efficacies (11). There is no clear guideline on which anticancer therapy should be chosen.

The histoculture drug response assay (HDRA) is a test that evaluates chemosensitivity to a given chemotherapy agent in vitro before treatment is initiated, using tumor tissue obtained during surgery to determine the appropriate drug of choice (12). The advantages of this assay are its short duration and drug delivery similar to the physiological condition due to the maintenance of cell-to-cell or cell-to-substrate interaction and preservation of physiological tumor cell structure during the test (12-14). The accuracy of HDRA was reported to be between $74-92.1 \%$ in cancer of the head and neck, stomach, ovaries, and colon (12-17). However, with the pre-established HDRA method, only chemosensitivity to first-line therapies can be tested. Hence, once chemoresistance develops, it is impossible to search for alternative chemotherapies. Furthermore, if cancer recurs after the initial surgery and adjuvant chemotherapy, it is then difficult to obtain cancer tissue. To improve upon this issue, at our Center, we pertinently applied an in vitro chemosensitive assay based on HDRA: the integrative tumor-response assay (ITRA; Patent No. 10-1046883; the Korean Intellectual Property Office, Seoul, Korea), which was developed by Kim and Moon (18). ITRA is a new in vitro tumor chemosensitivity assay, performed under near-physiological conditions, that tests for sensitivity not only to first-line chemotherapy agents, but also 
second-line agents that can be used in cases with chemoresistance to first-line drugs, using tumor specimens obtained during the initial surgery. ITRA is theoretically based on the HDRA procedure, with methods similar to those used in previous studies at our center $(15,16)$.

The current study aimed to evaluate the chemosensitivity of primary EOC in patients via ITRA using tumor tissue and to examine the real-world clinical outcomes.

\section{Patients and Methods}

Patients. This study was conducted with approval of the Institutional Review Board of Asan Medical Center (IRB number: 2012-0222). Forty-four patients with EOC were prospectively enrolled between March 2015 and December 2017. Informed consent was obtained from all patients. We included patients for whom we expected to use adjuvant chemotherapy during surgery, those with a histological diagnosis of epithelial carcinoma of the ovaries after primary cytoreductive surgery, those with International Federation of Obstetrics and Gynaecology (FIGO) stage IIIA to IVb cancer postoperatively, those with an Eastern Cooperative Oncology performance status $\leq 2$, and $\leq 80$ years old. Patients with a history of chemotherapy or radiotherapy preoperatively were excluded.

Primary cytoreductive surgery. All patients underwent complete staging surgery as the initial treatment. The complete staging surgeries included total abdominal hysterectomy, bilateral salphingo-oophorectomy, total omentectomy, pelvic and para-aortic lymphadenectomy, and tumorectomy of metastatic lesions. In cases with confirmed tumor invasion, resection surgery of tumor-invaded organs, such as colectomy, splenectomy, or liver segmentectomy, was also performed.

Chemotherapy selection. All patients received adjuvant chemotherapy with paclitaxel and carboplatin postoperatively. If combination therapy was unsuitable because of severe side-effects, underlying disease, or advanced age, single-agent paclitaxel or carboplatin therapy was given. After the initial chemotherapy cycle, patients with a platinum-free interval (PFI) of $\geq 12$ months were considered to have platinum-sensitive recurrence; those with $\mathrm{PFI} \geq 6$ months but $\leq 12$ months had partially platinum-sensitive recurrence, and those with PFI $<6$ months had platinum-resistant recurrence (19). Paclitaxel or carboplatin was chosen as the second-line chemotherapy for patients with platinum-sensitive recurrence. The main chemotherapy regimen was chosen based on ITRA results in patients with partially platinum-sensitive disease or those with platinum-resistant recurrent lesions or disease progression during first-line chemotherapy. For second-line chemotherapy, drugs with sensitivity higher than the inhibition rate of first-line chemotherapy as per the ITRA results were chosen, considering the patient's underlying disease and side-effects.

All 44 patients in this study underwent primary cytoreductive surgery. Tumor specimens were collected intraoperatively for ITRA. All patients underwent adjuvant chemotherapy: 43 with paclitaxel and carboplatin and one with carboplatin only because of hypersensitivity to paclitaxel. The disease progressed in 11 patients during first-line chemotherapy cycle, and one refused to participate further. Fifteen patients showed recurrence after initial chemotherapy, five of whom had platinumsensitive recurrence. Ten patients had partially platinum-sensitive or platinum-resistant recurrence, two of whom refused to participate further and were lost to follow-up. Consequently, 18 patients underwent secondline chemotherapy based on the ITRA results (Figure 1).

ITRA procedure. (i) Tissue preparation: Approximately $200 \mathrm{mg}$ of tumor tissue was obtained to confirm EOC on frozen biopsies; tissue was washed three times in 1-2\% povidone-iodine (Besetine Solution, Hyundai Pharmaceuticals, Cheonan, Republic of Korea) and saline solution. The tissue was transported to our laboratory in tissue transport media (Hanks's balanced salt solution, $2 \%$ sodium bicarbonate, $10 \%$ fetal bovine serum (FBS), $1 \%$ penicillinstreptomycin, and $0.4 \%$ gentamycin) at $4{ }^{\circ} \mathrm{C}$.

The above tissue was then washed three times or more in phosphate-buffered saline (PBS), $1 \%$ penicillin-streptomycin, and $0.4 \%$ gentamycin. A sterile tissue clamp and scalpel were used to dissect away all non-cancerous parts. The remaining tissue was moved to a new Petri dish and cut into $0.5-1.0 \mathrm{~mm}^{3}$ pieces. Chemosensitivity was maintained under near physiological conditions by preserving the three-dimensional structure of the tissue rather than separating the cells.

The resected tissue was washed twice in an antibacterial culture solution [RPMI 1640 medium (Gibco, Grand Island, NY, USA), 2\% sodium bicarbonate, $10 \%$ FBS, $1 \%$ penicillin-streptomycin, $0.4 \%$ gentamycin, $1 \%$ tetracycline, and 10\% chloramphenicol (Carl Roth, Karlsruhe, Germany), $1 \times$ tetrazolium salt (MTS; Promega, Madison, WI, USA)] and cultured in a $\mathrm{CO}_{2}$ incubator for 2 hours at $37^{\circ} \mathrm{C}$. The surviving tissue was then stained with tetrazolium salt (MTS; Promega, Madison, WI, USA), preventing non-specific drug reactivity to dead cells. The stained and selected tissues were moved to a Petri dish and cultured under $\mathrm{CO}_{2}$ for 24 hours at $37^{\circ} \mathrm{C}$. Destained tissue samples of equal size were placed in a 96-well plate (TPP, Trasadingen, Switzerland) (Figure 2).

(ii) ITRA first-line chemotherapy: The first-line chemotherapy agent was instilled in the wells of primary and secondary reaction plates, and PBS, which was used to wash tissue, was instilled in the control well. The samples were cultured for 72 hours. All chemotherapy agents were refrigerated, and drugs to be used were prepared in advance according to their combinations.

(iii) Analysis of first-line chemotherapy well and instillation of a second-line chemotherapy agent: The culture solution instilled with the first-line chemotherapy agent was discarded, and $100 \mu \mathrm{l}$ of 3-(4,5dimethylthiazol-2-yl)-2,5-diphenyl tetrazolium bromide (MTT; Sigma, St. Louis, MO, USA) was instilled in the primary wells and cultured in a $\mathrm{CO} 2$ incubator for $3-4$ hours at $37^{\circ} \mathrm{C}$. The reaction plates in which the second-line chemotherapy agent was to be deposited were treated with an additional secondary chemotherapy agent and cultured for 48 hours. The culture solution treated with MTT in the first-line chemotherapy reaction plate was discarded, and $100 \mu$ of dimethyl sulfoxide (Amresco, Solon, OH, USA) was added, which was shaken for 1-2 $\mathrm{h}$ for destaining. The destained solution was moved to a new 96-well plate and analyzed with an absorption spectrometer (VersaMax, Sunnyvale, CA, USA) at measurement and reference wavelengths of 570 and $630 \mathrm{~nm}$ respectively.

(iv) Analysis of the second-line chemotherapy well: The reaction plates treated with the second-line chemotherapy agent were destained, followed by analysis with absorption spectrometry. Inhibition ratio (IR) of $30 \%$ or above was deemed positive for tumor cell chemosensitivity according to the following equation: IR $(\%)=(1-\mathrm{T} / \mathrm{C}) \times 100$

where $\mathrm{T}$ was the absorbance per gram of tumor tissue in the chemotherapy well and $\mathrm{C}$ was the absorbance per gram of tumor tissue in the control well. 


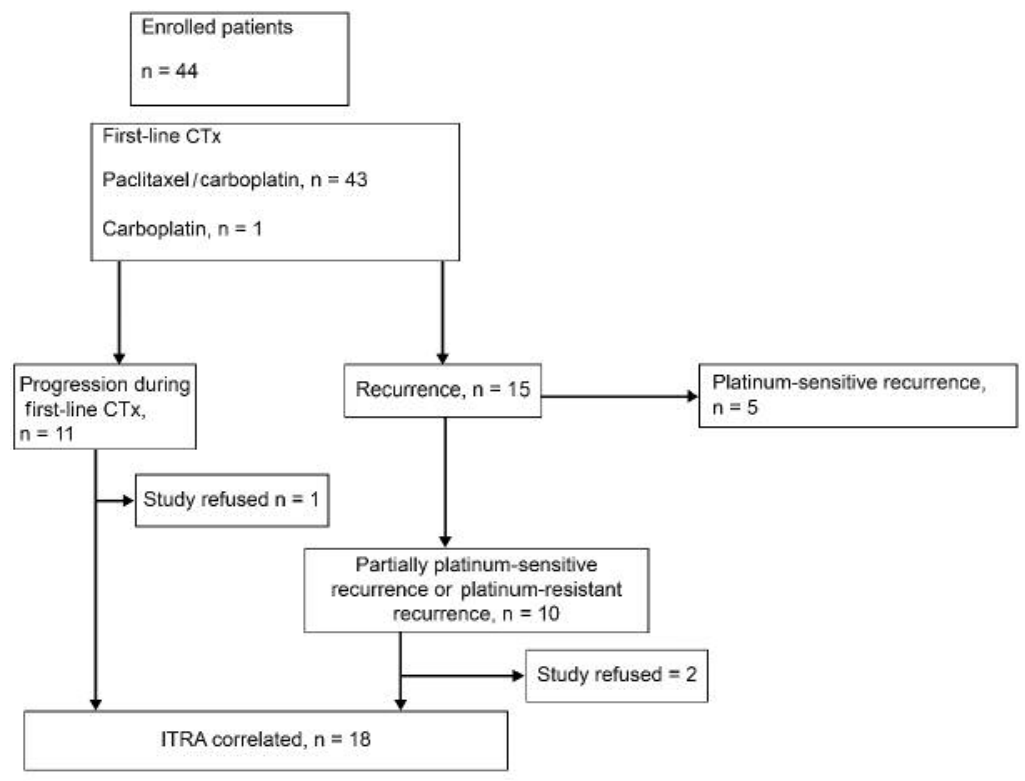

Figure 1. Selection of patients with advanced epithelial ovarian cancer. CTx: Chemotherapy; ITRA: integrative tumor-response assay.

(v) Investigated drug combinations and concentrations: Standard drug combinations available for clinical use, as per the revised criteria of the Korean Health Insurance Review and Assessment Service from September 2014, were used. Second-line combinations were also chosen among drugs approved for reimbursement at the time of the study. The concentrations of drugs for primary chemotherapy were $25 \mu \mathrm{g} / \mathrm{ml}$ carboplatin (Neoplatin; Boryung Pharmaceuticals, Seoul, Korea), 5 $\mu \mathrm{g} / \mathrm{ml}$ paclitaxel (Taxol; Bristol-Myers Squibb Pharmaceutical Korea, Seoul, Korea) for single therapy; and $25 \mu \mathrm{g} / \mathrm{ml}$ carboplatin with $5 \mu \mathrm{g} / \mathrm{ml}$ paclitaxel for combination. For single-regimen second-line drugs, 5 $\mu \mathrm{g} / \mathrm{ml}$ topotecan (Hycamtin; GlaxoSmithKline Korea, Seoul, Korea), 20 $\mu \mathrm{g} / \mathrm{ml}$ belotecan (Camtobell; Chong Keun Dang, Seoul, Korea), 50 $\mu \mathrm{g} / \mathrm{ml}$ gemcitabine (Gemzar; Lilly Korea, Seoul, Korea), $6 \mu \mathrm{g} / \mathrm{ml}$ doxorubicin (Ildong Adriamicin; Ildong Pharmaceuticals, Seoul, Korea), $250 \mu \mathrm{g} / \mathrm{ml}$ ifosfamide (Holoxan; Bukwang Pharmaceuticals, Seoul, Korea), $7.5 \mu \mathrm{g} / \mathrm{ml}$ vinorelbine (Navelbine; Bukwang Pharmaceuticals), and $50 \mu \mathrm{g} / \mathrm{ml}$ etoposide (EPS; Boryung Pharmaceuticals) were used.

Evaluation of tumor status. Tumor status was evaluated via computed tomography (CT) after three cycles of chemotherapy. CT was also performed whenever clinical signs of disease progression were noted. Cancer antigen 125 was measured during every cycle. According to the revised Response Evaluation Criteria in Solid Tumors (RECIST) guideline (version 1.1, 2009) (20), a complete response (CR) was disappearance of all target lesions, partial response (PR) was defined as a greater than $30 \%$ decrease in the sum of longest diameters of the target lesions without a new metastatic lesion, progressive disease (PD) was defined as a greater than $20 \%$ increase in the sum of longest diameters of the target lesions, and stable disease (SD) was neither a $\mathrm{PR}$ nor PD. The objective response rate (ORR) was defined as CR plus PR. Since the drug used for SD is continued until PD occurs in actual clinical settings, the clinical benefit response rate was defined as the sum of CR, PR and SD. The primary endpoint was the correlation between ITRA results and CR, seen with the ORR.

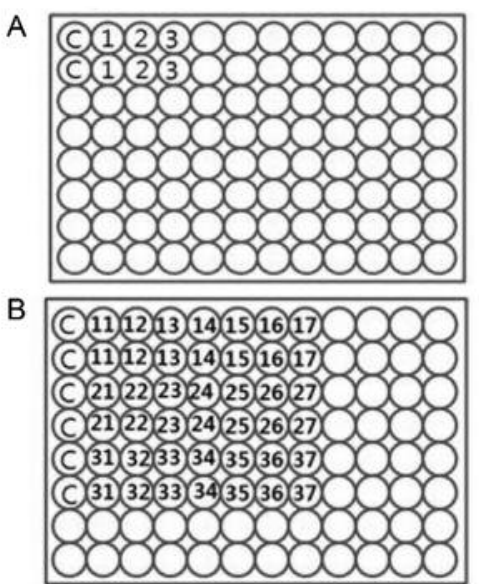

Figure 2. Integrative tumor-response assay. The arrangement of 96-well plates with first- (A) and second-line (B) chemotherapy agents for advanced epithelial ovarian cancer. A: C: PBS control, 1: carboplatin, 2: paclitaxel, 3: carboplatin+paclitaxel. B: Two-digit coding is used, the first digit represents the first-line chemotherapy: 1\#: Carboplatin, 2\#: paclitaxel, 3\#: carboplatin+paclitaxel; the second digit represents the second-line chemotherapy: \#1: topotecan, \#2: belotecan, \#3: gemcitabine, \#4: doxorubicin, \#5: ifosfamide, \#6: vinorelbine, \#7: etoposide. For example, 11 represents first-line therapy with carboplatin and second-line with topotecan, while 25 represents first-line therapy with paclitaxel and second-line with ifosfamide.

Statistical analysis. Descriptive statistics were used to analyze patient clinicopathological characteristics. To analyze the performance of ITRA, the sensitivity, specificity, accuracy, and negative and positive predictive values for predicting response were calculated, 95\% confidence intervals for these rates using the exact binomial method. 
Table I. Clinicopathological characteristics of patients, $n=44$.

\begin{tabular}{|c|c|}
\hline Characteristics & Value \\
\hline \multicolumn{2}{|l|}{ Age at initial diagnosis, years } \\
\hline Median (range) & $54(25-79)$ \\
\hline \multicolumn{2}{|l|}{ Follow-up period, months } \\
\hline Median (range) & $23.35(4-35.5)$ \\
\hline \multicolumn{2}{|l|}{ Body mass index, $\mathrm{kg} / \mathrm{m}^{2}$} \\
\hline Median (range) & $22.25(15.35-34.99)$ \\
\hline \multicolumn{2}{|l|}{ Parity, n } \\
\hline Median (range) & $2(0-3)$ \\
\hline \multicolumn{2}{|l|}{ Initial CA-125, U/ml } \\
\hline Median (range) & $1,003.95(30.6-9,834)$ \\
\hline \multicolumn{2}{|l|}{ FIGO stage, n (\%) } \\
\hline IIIa & $2(4.5 \%)$ \\
\hline IIIb & $5(11.4 \%)$ \\
\hline IIIc & $16(36.4 \%)$ \\
\hline IV & $21(47.7 \%)$ \\
\hline \multicolumn{2}{|l|}{ Surgical procedure, n (\%) } \\
\hline Debulking operation & $44(100 \%)$ \\
\hline \multicolumn{2}{|l|}{ Histological type, n (\%) } \\
\hline Serous & $37(84.1 \%)$ \\
\hline Other & $7(15.9 \%)$ \\
\hline \multicolumn{2}{|l|}{ Residual tumor, n (\%) } \\
\hline$<1 \mathrm{~cm}$ & $30(68.2 \%)$ \\
\hline$\geq 1 \mathrm{~cm}$ & $14(31.8 \%)$ \\
\hline \multicolumn{2}{|l|}{ Progression, n (\%) } \\
\hline During 1st-line chemotherapy & $11(25.6 \%)$ \\
\hline \multicolumn{2}{|l|}{ Recurrence, $\mathrm{n}(\%)$} \\
\hline No & $18(40.9 \%)$ \\
\hline Yes & $15(34.1 \%)$ \\
\hline \multicolumn{2}{|l|}{ Death from disease, $\mathrm{n}(\%)$} \\
\hline No & $36(81.8 \%)$ \\
\hline Yes & $8(18.2 \%)$ \\
\hline
\end{tabular}

A Chi-squared test was used for a cross-table analysis to compare categorical variables. A $p$-value less than 0.05 was deemed statistically significant. All statistical analyses were performed with SPSS software, version 21.0 (SPSS, IBM Corp., Armonk, NY, USA).

\section{Results}

Forty-four patients met this study's criteria. The median followup period was 23.35 months (range=4-35.5 months). The clinicopathological characteristics of patients are summarized in Table I. All patients underwent primary cytoreductive surgery. Among them, $84.1 \%$ were diagnosed with the serous type of ovarian carcinoma, and $68.2 \%$ with a residual tumor size of less than $1 \mathrm{~cm}$ underwent optimal debulking surgery. Eleven patients $(25.6 \%)$ showed tumor progression during the first chemotherapy cycle after surgery. Fifteen patients (34.1\%) had recurrent tumor after adjuvant chemotherapy, and eight patients $(18.2 \%)$ died of their disease.

Table II shows the mean IR for the in vitro chemosensitivity of ITRA. The mean IR when paclitaxel and carboplatin were used as first-line therapy was 53\% (range=19-79\%).
Table II. Average tumor inhibition rate (IR) in the integrative tumorresponse assay.

\begin{tabular}{lcc}
\hline Chemotherapy line & Drug & Mean IR (range), \% \\
\hline First & Paclitaxel-carboplatin & $53(19-79)$ \\
Second & Topotecan & $66(12-96)$ \\
& Belotecan & $67(25-97)$ \\
& Gemcitabine & $63(20-95)$ \\
& Adriamycin & $68(35-95)$ \\
& Ifosfamide & $67(10-95)$ \\
& Vinorelbine & $57(11-91)$ \\
& Etoposide & $68(19-96)$ \\
\hline
\end{tabular}

Table III. Main second-line chemotherapy.

\begin{tabular}{ll}
\hline Drug & N (\%) \\
\hline Pegylated liposomal doxorubicin & $8(44.4)$ \\
Belotecan & $6(33.3)$ \\
Topotecan & $4(22.2)$ \\
\hline
\end{tabular}

Adriamycin and etoposide had the highest mean IR at $68 \%$ (range $=19-96 \%$ and $35-95 \%$, respectively) among the secondline chemotherapy agents.

Based on the results of ITRA, pegylated liposomal doxorubicin was the most frequently used second-line chemotherapy agent, followed by belotecan (Table III).

Table IV shows the treatment response to second-line chemotherapy. The ORR was $38.9 \%$. Four patients (22.2\%) showed a CR and three (16.7\%) had PRs in target lesions (according to the RECIST criteria). The clinical benefit response rate was $50 \%$, including two patients (11.1\%) with SD. The sensitivity of ITRA was $85.7 \%$, specificity was $18.2 \%$, negative predictive value was $66.7 \%$, positive predictive value was $40 \%$, the accuracy was $44.44 \%$ (Table V).

Advanced-stage tumors $(p=0.045)$ and serous-type tumors by histology $(p=0.005)$ were related to poor chemo-responsiveness towards first-line chemotherapy. No variables were significantly related to the patient's responsiveness towards the second-line chemotherapy.

\section{Discussion}

We evaluated patients with primary ovarian cancer in terms of chemosensitivity via ITRA using tumor tissue and attempted to determine the real-world applicability of ITRAs. This study showed that ITRAs had acceptable applicability.

After the standard first-line treatment including surgery followed by platinum-based chemotherapy for advanced stages of ovarian cancer, patients showed high overall response rates 
Table IV. Treatment response to second-line chemotherapy.

\begin{tabular}{lcc}
\hline & \multicolumn{2}{c}{ Second-line chemotherapy $\mathrm{n}=18$} \\
\cline { 2 - 3 } & $\mathrm{n}$ & $\%$ \\
\hline Clinical response (RESCIST 1.1) & & \\
CR & 4 & 22.2 \\
PR & 3 & 16.7 \\
SD & 2 & 11.1 \\
PD & 9 & 50 \\
Objective response rate & & \\
CR+PR & 7 & 38.9 \\
Clinical benefit response rate & & \\
CR+PR+SD & 9 & 50 \\
\hline
\end{tabular}

CR: Complete response; PR: partial response; SD: stable disease.

Table V. Correlation between integrative tumor-response assay (ITRA) and clinical response to second-line chemotherapy in patients with recurrent ovarian cancer.

\begin{tabular}{lccc}
\hline & \multicolumn{3}{c}{ Clinical response, $\mathrm{n}$} \\
\cline { 2 - 4 } ITRA, n & Positive** $^{* *}$ & Negative & Total \\
\hline Positive* & 6 & 9 & 15 \\
Negative & 1 & 2 & 3 \\
Total & 7 & 11 & 18 \\
\hline
\end{tabular}

Sensitivity: $85.7 \%$, specificity: $18.2 \%$, negative predictive value: $66.7 \%$, positive predictive value: $40 \%$, accuracy: $44.44 \%$. $*$ Inhibition rate $\geq 30 \%$. **Complete response or partial response.

of $30-50 \%(21,22)$. However, the 10 -year relative survival rate was only $10-20 \%$, implying that most patients experience relapse, develop chemoresistance, and eventually die. Therefore, most patients are eligible for second-line chemotherapy (23). Considering the characteristics of ovarian cancer, generally agreed upon a first-line chemotherapy agent, and the high recurrence rate of tumors requiring a second-line agent, we developed ITRA for patients with advanced EOC.

ITRA is not a simple repetition of HDRA, but an evaluation of chemosensitivity towards second-line therapy in tumor cells that survived the first-line therapy. The theory of ITRA is based on the assumption that the genetic traits of in vitro and in vivo tumor cells in patients with recurrent cancer would be the same (18).

In this study, the ORR and CRs were similar to or slightly higher than those reported in previous studies (24-27). According to a multivariate analysis by Blackledge $e t$ al. of 92 patients enrolled in five phase II chemotherapy trials, the response rate to second-line chemotherapy was about $10 \%$ in patients with a treatment-free interval (TFI) $\leq 6$ months and $29 \%$ in those with TFI of 7-12 months (28). Most patients initially undergo treatment with platinum-based chemotherapy; thus, the PFI and TFI are often considered equivalent in patients with recurrent ovarian cancer. According to Pujade-Lauraine et al., in patients with a TFI $<12$ months, the response rates to secondline chemotherapy ranged from $24-35 \%$ (24).

In this study, the sensitivity of ITRA was high and its specificity was low. Sensitivity is considered equivalent to the true-positive rate (29), and the high sensitivity in this study signifies that the chemotherapy agent selected based on ITRA results had a high rate of clinical efficacy. Therefore, ITRA may help with screening to choose the second-line drug. The specificity was relatively low, and this may have been affected by the fact that all three patients who refused to participate further in the study had a low IR to second-line therapy by ITRA.

In a study on metastatic colorectal cancer, the sensitivity of ITRA was $44.4 \%$, specificity $75 \%$, and accuracy $61.9 \%$ (18). The difference between metastatic colorectal cancer and ovarian cancer might be due to the characteristics of each cancer type. As mentioned by Yoon et al. in a study on ITRA, the use of ITRA is limited because in vivo conditions, including circulating tumor cells or cancer stem cells related to chemoresistance, cannot be reproduced completely (18).

This study has certain limitations. The first is the choice of second-line chemotherapy agents. The seven drugs chosen as second-line agents were those used clinically approved by the Korean government at the time ITRA was developed. At that time, the Korean government had not yet approved pegylated liposomal doxorubicin, which is now widely used. After it was included in the reimbursement scheme, pegylated liposomal doxorubicin was chosen instead of doxorubicin, but this was not updated in the ITRA protocol. Additionally, combination therapy including bevacizumab is now eligible for reimbursement as a first-line chemotherapy regimen. Cisplatin is also sometimes used in combination with other drugs. Adding combination therapies to ITRA would provide more useful and significant results. The short-term follow-up period and small sample size are also limitations of this study. According to our previous study, there was a significant difference in recurrence and progression-free survival between paclitaxel/carboplatin-sensitive and resistant patients by HDRA (16). In this study, the relationship between the result of first stage of ITRA and such outcomes was difficult to establish because of the short-term follow-up period and small sample size. However, this study is significant because as far as we are aware it is the first prospective study on ITRA for patients with EOC. Furthermore, it showed the applicability of ITRA, since the response rate to second-line therapy, chosen based on the ITRA results, was similar or slightly higher than that seen in previous studies (24-27), and it has a high sensitivity. Studies with a larger sample size and longer follow-up periods should be performed to confirm the efficacy of ITRA. 
In conclusion, although ITRA has a relatively low accuracy and specificity, it is feasible and applicable and may be a useful tool to help physicians choose second-line chemotherapy regimens in patients with advanced EOC.

\section{References}

1 Ferlay J, Soerjomataram I, Dikshit R, Eser S, Mathers C, Rebelo M, Parkin DM, Forman D and Bray F: Cancer incidence and mortality worldwide: sources, methods and major patterns in GLOBOCAN 2012. Int J Cancer 136: E359-386, 2015.

2 Siegel RL, Miller KD and Jemal A: Cancer statistics, 2017. CA Cancer J Clin 67: 7-30, 2017.

3 Webb PM and Jordan SJ: Epidemiology of epithelial ovarian cancer. Best Pract Res Clin Obstet Gynaecol 41: 3-14, 2017.

4 Jessmon $\mathrm{P}$, Boulanger $\mathrm{T}$, Zhou $\mathrm{W}$ and Patwardhan $\mathrm{P}$ : Epidemiology and treatment patterns of epithelial ovarian cancer. Expert Rev Anticancer Ther 17: 427-437, 2017.

5 Gadducci A, Cosio S, Conte PF and Genazzani AR: Consolidation and maintenance treatments for patients with advanced epithelial ovarian cancer in complete response after first-line chemotherapy: A review of the literature. Crit Rev Oncol Hematol 55: 153-166, 2005.

6 Markman M, Liu PY, Wilczynski S, Monk B, Copeland LJ, Alvarez RD, Jiang C, Alberts D, Southwest Oncology Group and Gynecologic Oncology Group: Phase III randomized trial of 12 versus 3 months of maintenance paclitaxel in patients with advanced ovarian cancer after complete response to platinum and paclitaxel-based chemotherapy: A Southwest Oncology Group and Gynecologic Oncology Group trial. J Clin Oncol 21: 2460-2465, 2003.

7 Stuart GC: First-line treatment regimens and the role of consolidation therapy in advanced ovarian cancer. Gynecol Oncol 90: S8-15, 2003.

8 Rose PG, Tian C and Bookman MA: Assessment of tumor response as a surrogate endpoint of survival in recurrent/ platinum-resistant ovarian carcinoma: a Gynecologic Oncology Group study. Gynecol Oncol 117: 324-329, 2010.

9 Ledermann JA, Raja FA, Fotopoulou C, Gonzalez-Martin A, Colombo N, Sessa C and ESMO Guidelines Working Group: Newly diagnosed and relapsed epithelial ovarian carcinoma: ESMO Clinical Practice Guidelines for diagnosis, treatment and follow-up. Ann Oncol 24(Suppl 6): vi24-32, 2013.

10 Ledermann JA and Raja FA: Clinical trials and decision-making strategies for optimal treatment of relapsed ovarian cancer. Eur J Cancer 47(Suppl 3): S104-115, 2011.

11 Cancer Genome Atlas Research Network: Integrated genomic analyses of ovarian carcinoma. Nature 474: 609-615, 2011.

12 Hoffman RM: Three-dimensional histoculture: Origins and applications in cancer research. Cancer Cells 3: 86-92, 1991.

13 Furukawa T, Kubota T and Hoffman RM: Clinical applications of the histoculture drug response assay. Clin Cancer Res 1: 305-311, 1995.

14 Hasegawa Y, Goto M, Hanai N, Ijichi K, Adachi M, Terada A, Hyodo I, Ogawa T and Furukawa T: Evaluation of optimal drug concentration in histoculture drug response assay in association with clinical efficacy for head and neck cancer. Oral Oncol 43: 749-756, 2007.

15 Yoon YS, Kim CW, Roh SA, Cho DH, Kim GP, Hong YS, Kim TW, Kim MB and Kim JC: Applicability of histoculture drug response assays in colorectal cancer chemotherapy. Anticancer Res 32: 3581-3586, 2012.
16 Jung PS, Kim DY, Kim MB, Lee SW, Kim JH, Kim YM, Kim YT, Hoffman RM and Nam JH: Progression-free survival is accurately predicted in patients treated with chemotherapy for epithelial ovarian cancer by the histoculture drug response assay in a prospective correlative clinical trial at a single institution. Anticancer Res 33: 1029-1034, 2013.

17 Hoffman RM: Clinical correlation of the histoculture drug response assay in gastrointestinal cancer. Methods Mol Biol 1760: 61-72, 2018.

18 Yoon YS, Kim CW, Roh SA, Cho DH, Kim TW, Kim MB and Kim JC: Development and applicability of integrative tumor response assays for metastatic colorectal cancer. Anticancer Res 37: 1297-1303, 2017.

19 Colombo N: Optimising the treatment of the partially platinumsensitive relapsed ovarian cancer patient. EJC Suppl 12: 7-12, 2014.

20 Eisenhauer EA, Therasse P, Bogaerts J, Schwartz LH, Sargent D, Ford R, Dancey J, Arbuck S, Gwyther S, Mooney M, Rubinstein L, Shankar L, Dodd L, Kaplan R, Lacombe D and Verweij J: New response evaluation criteria in solid tumours: Revised RECIST guideline (version 1.1). Eur J Cancer 45: 228-247, 2009.

21 Ozols RF, Bundy BN, Greer BE, Fowler JM, Clarke-Pearson D, Burger RA, Mannel RS, DeGeest K, Hartenbach EM and Baergen R: Phase III trial of carboplatin and paclitaxel compared with cisplatin and paclitaxel in patients with optimally resected stage III ovarian cancer: A Gynecologic Oncology Group study. J Clin Oncol 21: 3194-3200, 2003.

22 du Bois A, Luck HJ, Meier W, Adams HP, Mobus V, Costa S, Bauknecht T, Richter B, Warm M, Schroder W, Olbricht S, Nitz U, Jackisch C, Emons G, Wagner U, Kuhn W and Pfisterer J: A randomized clinical trial of cisplatin/paclitaxel versus carboplatin/paclitaxel as first-line treatment of ovarian cancer. J Natl Cancer Inst 95: 1320-1329, 2003.

23 Rose PG, Monk BJ, Provencher D, Hartney J, Legenne P and Lane S: An open-label, single-arm phase II study of intravenous weekly (days 1 and 8) topotecan in combination with carboplatin (day 1) every 21 days as second-line therapy in patients with platinum-sensitive relapsed ovarian cancer. Gynecol Oncol 120: 38-42, 2011.

24 Colombo $\mathrm{N}$ and Gore M: Treatment of recurrent ovarian cancer relapsing 6-12 months post platinum-based chemotherapy. Crit Rev Oncol Hematol 64: 129-138, 2007.

25 Fung-Kee-Fung M, Oliver T, Elit L, Oza A, Hirte HW and Bryson P: Optimal chemotherapy treatment for women with recurrent ovarian cancer. Curr Oncol 14: 195-208, 2007.

26 Pujade-Lauraine E and Combe P: Recurrent ovarian cancer. Ann Oncol 27(Suppl 1): i63-i65, 2016.

27 Herzog TJ: Update on the role of topotecan in the treatment of recurrent ovarian cancer. Oncologist 7(Suppl 5): 3-10, 2002.

28 Blackledge G, Lawton F, Redman C and Kelly K: Response of patients in phase II studies of chemotherapy in ovarian cancer: Implications for patient treatment and the design of phase II trials. Br J Cancer 59: 650-653, 1989.

29 Lutkenhoner B and Basel T: Predictive modeling for diagnostic tests with high specificity, but low sensitivity: A study of the glycerol test in patients with suspected Meniere's disease. PLoS One 8: e79315, 2013. 